\title{
PHOTOMETRIC PROPERTIES AND METALLICITY OF V1719 CYGNI
}

\author{
Chulhee Kim ${ }^{1}$, And A. V. Yushchenko ${ }^{2}$ \\ ${ }^{1}$ Division of Science Education, Institute of Fusion Science, Chonbuk National University, Chonju 561-756, Korea \\ E-mail : ckim@chonbuk.ac.kr \\ ${ }^{2}$ Odessa Astronomical Observatory, Odessa National University, Park Shevchenko, Odessa, 65014, Ukraine \\ E-mail : avyushchenko@gmail.com \\ (Received March 18, 2011; Revised April 08, 2011; Accepted April 12, 2011)
}

\begin{abstract}
We collect 24 times of light maxima data from sources in the literature, unpublished data and open databases, and investigate the variations of the observed and calculated $(\mathrm{O}-\mathrm{C})$ values for light maxima of V1719 Cyg. We found no evidence of the variations in the $(\mathrm{O}-\mathrm{C})$ values. We estimate the effective temperature and surface gravity using both the Kurucz and MARCS/SSG grids for different metallicity values $[\mathrm{A} / \mathrm{H}]=0.0$ and +0.5 for V1719 Cyg. It is confirmed that the temperature is almost the same, but, in the case of surface gravity, the MARCS/SSG grid gives the value closest to that obtained from the period-gravity relation derived by using the pulsation-evolution theory. We obtain two spectra of V1719 Cyg from spectroscopic observation which permitted us to find the effective temperature and the surface gravity of the star directly. We estimate the metallicity and it is found that the abundance of iron is equal to the solar value.
\end{abstract}

Key words : stars: oscillation — stars: variables $(\delta$ Scuti $)$ - stars: individual $($ V1719 Cyg $)$ - stars: metallicity

\section{INTRODUCTION}

V1719 Cyg $(H D 200925),\left(\alpha=21^{\mathrm{h}} 02^{\mathrm{m}} 56^{\mathrm{s}}, \delta=\right.$ $+50^{\circ} 35$ ! $\left.15(2000),\langle\mathrm{V}\rangle=8^{\mathrm{m}} .11\right)$, was identified as a variable star by Bedolla \& Pena (1979) through observations on 4 nights with a $\mathrm{V}$ filter. Since then, many more observations have been carried out using the UBV and uvby $\beta$ color systems (see Pena et al. 2002 for earlier studies and references therein).

Mantegazza \& Poretti (1986) carried out a period analysis of three previous data sets: Poretti (1984) in UBV, Bedolla \& Pena (1979) in V, and Padalia \& Gupta (1982). They determined the existence of two periods, including a definite one at 0.267298 days and a possible second period at 0.2138 days. The ratio of these two periods is 0.800 , which matches the theoretically predicted period ratio by Cox et al. (1984).

Cox et al. (1984) show that this ratio can be possible for the fundamental and first overtone radial modes in correspondence with helium settling within the envelope of a $\delta$ Scuti star. In fact, it has been known that high metallicity and helium settling can be accounted for by the process of diffusion in Am/Fm stars. With regard to this, Poretti and Antonello (1988, hereafter PA88) argued for the possibility of the fundamental and first overtone modes indirectly by considering the color index and luminosity which place this star near the cool edge of the instability strip. Cox et al. (1979a,b) already proposed that a star near the instability strip

Corresponding Author: C. Kim may pulsate in spite of low helium content in its envelope and high metallicity. PA88 also pointed out that the period $p_{1}=0.26$ is rather long for High Amplitude $\delta$ Scuti (HADS) stars and that this period cannot correspond to the first overtone mode.

Radial modes of the first and second periods were directly confirmed by Kim et al. (1993) by adopting the method developed by Watson (1988) who had outlined a format for the comparison of observational light and color data with model predictions on an amplitude ratio versus phase difference plane. They also confirmed the fundamental and first overtone modes by adopting the method developed by Fitch (1981) which is applicable when the pulsation constant strongly depends on the pulsation mode $(\mathrm{n}, \mathrm{l})$.

It was first noticed by Joner and Johnson (1985) that the light curve of V1719 Cyg is very peculiar. The main peculiarity is the asymmetry with the descending branch steeper than the ascending branch. They also found that V1719 Cyg shows a definite variation of $m_{1}$ with temperature, but this variation is opposite to the variation predicted by Crawford (1979). On the contrary, by using the one zone model proposed by Stellingwerf (1972), Antonello (1991) obtained an interesting result concerning the inverse variation of the $m_{1}$-index and he argued that the cool stars with mildly rich atmospheres such as V1719 Cyg can show this kind of peculiarity.

Pena et al. (2002) observed V1719 Cyg using the uvby $\beta$ filters and estimated the atmospheric param- 
Table 1.

Light maxima of V1719 Cyg.

\begin{tabular}{crrc}
\hline \hline HJD & Cycle & O-C & Source \\
\hline 3776.7150 & -1629 & -.0028 & $\mathrm{BP}$ \\
3776.8350 & -1629 & 0.1172 & $\mathrm{PG}$ \\
3777.7880 & -1625 & 0.0010 & $\mathrm{BP}$ \\
3778.7130 & -1622 & 0.1241 & $\mathrm{PG}$ \\
3779.6590 & -1618 & 0.0010 & $\mathrm{BP}$ \\
3779.7760 & -1618 & 0.1180 & $\mathrm{PG}$ \\
4164.2950 & -179 & -.0038 & $\mathrm{PG}$ \\
4173.1180 & -146 & -.0016 & $\mathrm{PG}$ \\
4178.1930 & -127 & -.0053 & $\mathrm{PG}$ \\
4212.1510 & 0 & 0.0060 & $\mathrm{PG}$ \\
4518.7310 & 1147 & -.0040 & $\mathrm{GF}$ \\
5544.8850 & 4986 & -.0042 & $\mathrm{JJ}$ \\
5546.5010 & 4992 & 0.0080 & $\mathrm{EP}$ \\
5559.5920 & 5041 & 0.0015 & $\mathrm{EP}$ \\
5560.3960 & 5044 & 0.0036 & $\mathrm{EP}$ \\
5574.5640 & 5097 & 0.0048 & $\mathrm{EP}$ \\
5582.8530 & 5128 & 0.0076 & JJ \\
5583.9210 & 5132 & 0.0064 & JJ \\
5593.8030 & 5169 & -.0016 & JJ \\
5626.6890 & 5292 & 0.0068 & JJ \\
5627.7590 & 5296 & 0.0076 & JJ \\
5952.7770 & 6512 & -.0078 & JJ \\
6272.7460 & 7709 & 0.0063 & JJ \\
6281.8190 & 7743 & -.0088 & JJ \\
\hline & & &
\end{tabular}

eters. Their results of $[\mathrm{Fe} / \mathrm{H}]=1.020, T_{\text {eff }}=6400$ 7100 Kand $\log g=3.1$ are quite different from the values $(0.456-0.664,7470 \mathrm{~K}, 3.87)$ obtained by Johnson \& Joner (1986, hereafter JJ86). An exhaustive analysis of the photometry is required to determine the atmospheric parameters as accurately as possible. Especially, spectroscopic determination of metallicity is necessary.

Given the potential significance that a complete study of V1719 Cyg is likely to have, we have undertaken spectroscopic observations of high resolution in order to study the atmospheric parameters and metallicity. We also analyzed the O-C diagram to study the period variation and, in addition, we have tried to determine $T_{\text {eff }}$ and $[\mathrm{Fe} / \mathrm{H}]$ using the MARCS/SSG atmospheric model (Gustafsson et al. 1975; Bell, Paltoglou, \& Tripicco 1994) which has been rarely adopted for the study of HADS. These results are compared with those obtained from the Kurucz grids (1993, 1995).

\section{PHOTOMETRIC INVESTIGATIONS}

We began our analysis by plotting the $\mathrm{O}-\mathrm{C}$ diagram with 24 times of light maxima obtained from the literature as shown in Table 1 . We calculated the expected times of maximum light by applying the following equation derived by Poretti (1984),

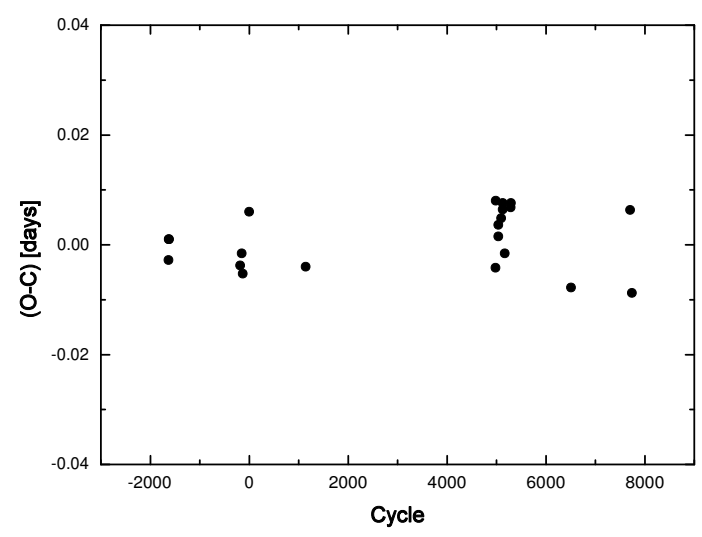

Fig. 1. - Difference between observed times of maximum light and times of maximum light calculated from Eq. (2).

$$
\mathrm{HJD}_{\max }=2444212.145+0.267298 \cdot E .
$$

On the basis of the above epoch and period values, we deduced the following ephemeris using all data points which covers a span of almost 8 years except for the three data points $2443776.835,2443778.788$, and 2443779.776 having larger $\mathrm{O}-\mathrm{C}$ values.

$$
\mathrm{HJD}_{\max }=2444212.145(2)+0.2672973(5) \cdot E .(2)
$$

Fig. 1 shows a plot of the differences between the observed and the calculated times of maximum light (O-C) versus the calculated cycle. Three outliers corresponding to the cycle of $-1629,-1622$ and -1618 with $\mathrm{O}-\mathrm{C}$ values of $+0.1172,+0.1241$, and 0.1180 were not included in the plot. At a first glance, there is no systematic variation, which means that the period has been stable for more than eight years.

Until this time, the Kurucz model has been widely used to estimate the effective temperature and surface gravity. Time to time calibration by Moon \& Dworetsky (1985) was adopted. This is based on the Kurucz model too. A new grid of theoretical color indices for the uvby photometric system has been derived from MARCS model atmospheres and SSG code (Bell, Paltoglu, \& Tripicco 1994) by Clem et al. (2004).

Because this model has never been used to determine $T_{\text {eff }}$ and $\log g$ for HADS stars, we adopted this model for V1719 Cyg and the results were compared with those from P02 where the Kurucz model was used. Since $[\mathrm{Fe} / \mathrm{H}]$ value was estimated to be $0.456-0.664$ by JJ and 1.020 by Pena, and since the maximum $[\mathrm{Fe} / \mathrm{H}]$ value in the MARCS model is 0.5 , we determined $[\mathrm{Fe} / \mathrm{H}]$ for both 0.0 and 0.5 . Photometric uvby $\beta$ 
Table 2.

$T_{\text {eff }}$ and $\log g$ from the Kurucz and MARCS grids.

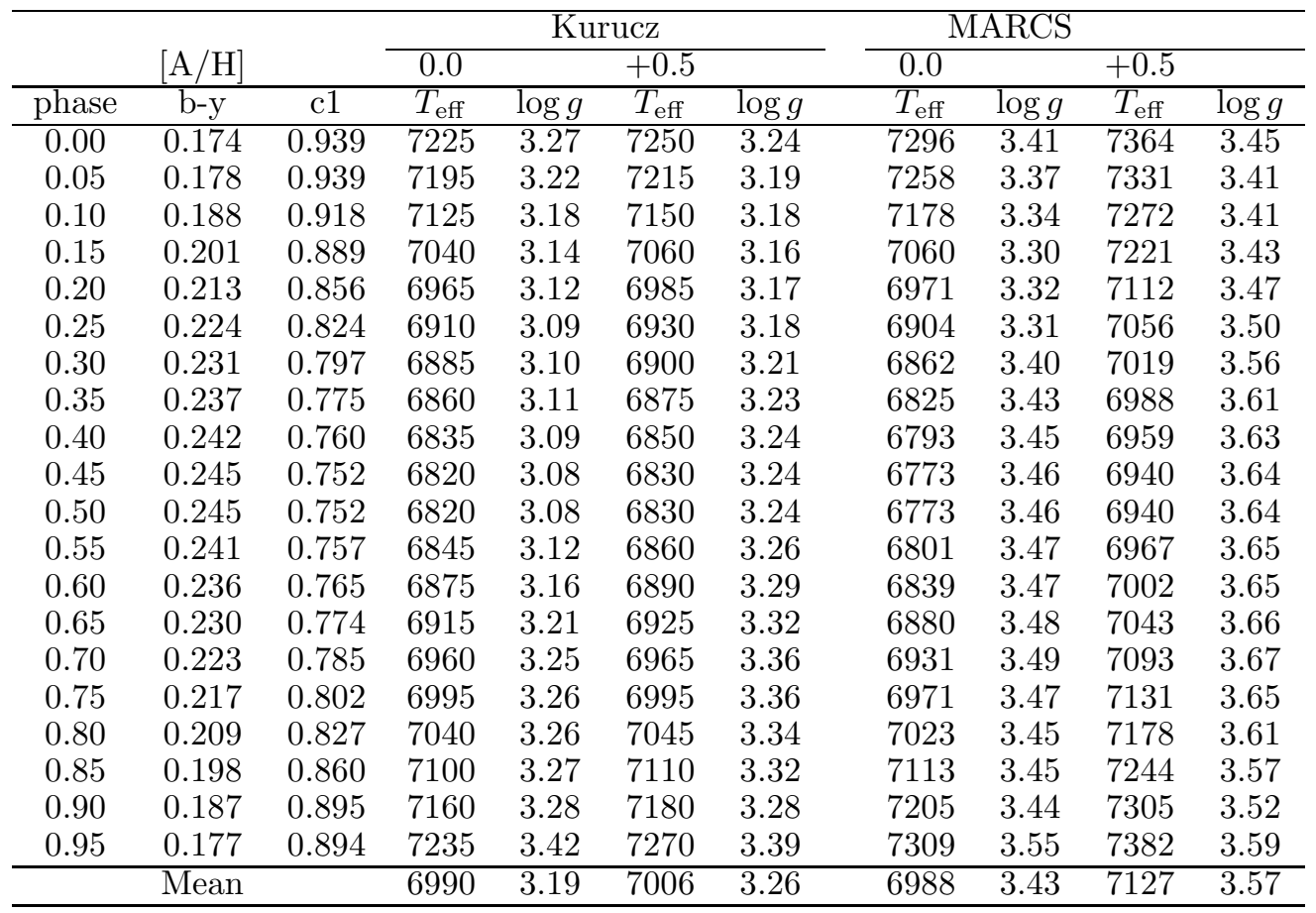

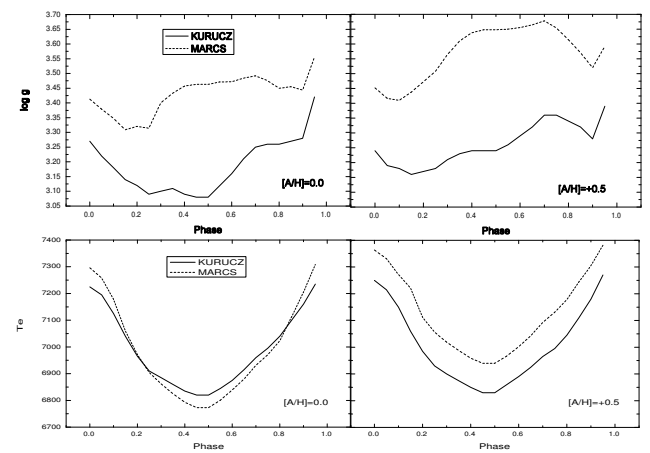

Fig. 2.- The effective temperatures and surface gravities of V1719 Cyg as a function of the phase of pulsation. The values found from Kurucz and MARCS grids of atmosphere models are shown.

data was taken from Kim et al. (1993) who separated the light curve of V1719 Cyg corresponding to the first and second periods.

The result is presented in Table 2 and Fig. 2. The second and third columns correspond to the photometric indices in the uvby color system. At the light minimum region, the MARCS model gives hotter $T_{\text {eff }}$. For $[\mathrm{Fe} / \mathrm{H}]=+0.5$, the KURUCZ model gives around $100 \mathrm{~K}$ hotter compared to MARCS model for all the phases but, the mean $T_{\text {eff }}$ is almost the same for both. However, in the case of surface gravity, the difference between the two models is large. The $\log g$ values are around 0.4 smaller over the whole cycle for the Kurucz model. It seems that the different metallicity causes the large difference of $T_{\text {eff }}$ and $[\mathrm{Fe} / \mathrm{H}]$ by Pena et al. (2002).

One can estimate $\log g$ from the $\left(\log g, \log P_{0}\right)$ diagram for HADS stars given by Andreasen (1983). However, the surface gravities for dwarf Cepheids are expected to be overestimated by about 0.1 in $\log g$ (Andreasen 1983). The calibration of $\log g$ from uvby $\beta$ photometry was based upon standard stars (ordinary rotational velocity), while the dwarf Cepheids are slow rotators with $\mathrm{v} \sin \mathrm{i}<20 \mathrm{~km} / \mathrm{s}$ (McNamara 1985), and therefore, the $\log g$ value needs to be corrected for dwarf cepheids. However, it is difficult to understand why $\log g$ values estimated from two different atmospheric models are different for all phases.

For the first period $0.267, \log g$ is about 3.4 which is value is close to 3.43 obtained from the MARCS model with $[\mathrm{Fe} / \mathrm{H}]=0.0$. Kurucz results for both metallicities are about 0.2 smaller. Hence, if the period-gravity relation by Andreasen $(1983,1987)$ is appropriate for V1719 Cyg and this variable star can be classified as a HADS star, then the MARCS model is better for estimating the surface gravity. 


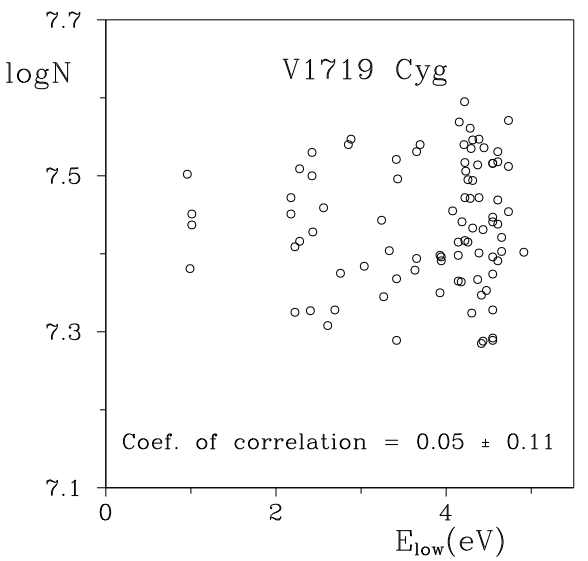

Fig. 3. - The dependence of iron abundances derived from the lines of neutral iron in the spectrum of V1719 Cyg vs. the energies of low level of these lines.

\section{SPECTROSCOPIC INVESTIGATIONS}

In order to estimate the metallicity of V1719 Cyg two high-resolution spectra of the star were obtained using the Bohyunsan Echelle Spectrograph (BOES) fed by the $1.8 \mathrm{~m}$ telescope of the Bohyunsan Optical Astronomy Observatory in Korea. The resolving power of the spectrum is $\mathrm{R}=45,000$, the signal to noise ratio is about $\mathrm{S} / \mathrm{N}=100$ in the red spectral region, the wavelength coverage is from 3800 to 9600 angstroms and the Julian dates of observations are 2454740.115 and 2454740.187. The exposure times for both spectra are equal to 30 minutes.

Our reduction of the Echelle spectrum was performed using both the IRAF (Tody 1986) and DECH codes (Galazutdinov 1992). The programs allowed us to perform all the standard procedures for CCD spectra processing and analyzing. The wavelength scale was constructed on the basis of a global polynomial of the form described in Galazutdinov et al. (2000). IRAF was used for image processing and the extraction of spectra from two-dimensional images. Further data processing was performed using the DECH and URAN (Yushchenko 1998) codes. Using parameters $T_{\text {eff }}=7000 \mathrm{~K}, \log g=3.5,[\mathrm{Fe} / \mathrm{H}]=0.0$ and 0.5 , which are between the parameters derived by Pena et al. (2002) and JJ86, the synthetic spectrum was calculated for the whole observed wavelength range. We used $\mathrm{Ku}-$ rucz (1993) SYNTHE code to determine this. Line data were taken from the Kurucz $(1993,1995)$ data base, Hirata \& Horaguchi (1995) line list, Morton (2000), the DREAM data base (Biemont et al. 2002), the VALD database (Piskunov et al. 1995), Fuhr \& Wiese (2006), and other sources.

Synthetic spectrum helps to place the continuum level and to identify the unblended lines of neutral and ionized iron. We measured the equivalent widths of these lines in the spectrum of V1719 Cyg.

The equivalent widths obtained were used to find

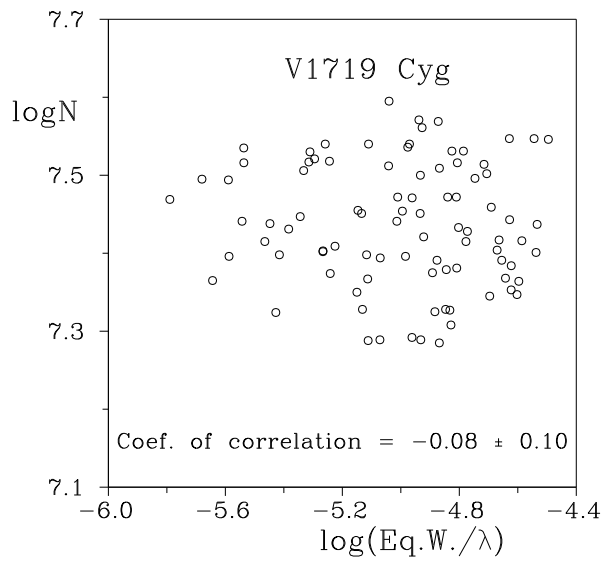

Fig. 4. - The dependence of iron abundances derived from the lines of neutral iron in the spectrum of V1719 Cyg vs. the equivalent widths $(\log ($ equivalent widths $/ \lambda)$ ) of these lines.

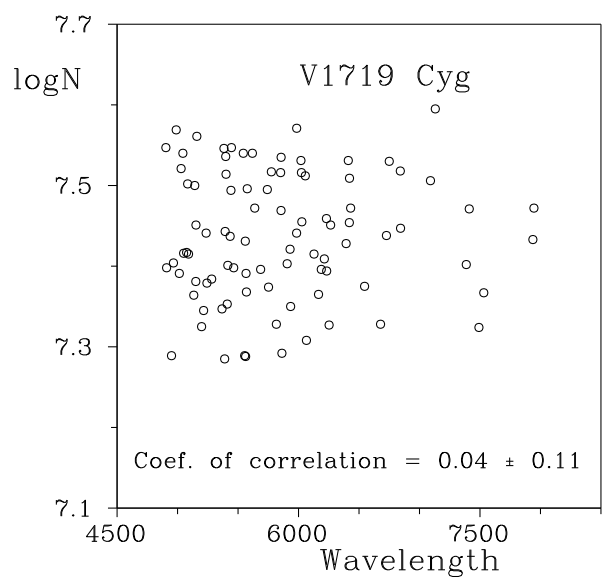

Fig. 5. - Similar to Figs. 3 and 4, but the argument is the wavelength.

values of the effective temperature, surface gravity, microturbulence and iron abundance directly from the observed spectrum. We used the method developed by Yushchenko et al. (1999) and described in more details in Gopka et al. (2004). This was helpful in our recent investigations of stars of different types (Yushchenko et al. 2005; Lee et al. 2006; Kim et al. 2007; Yushchenko et al. 2010).

Briefly, this method finds the iron abundances for a grid of models with different values of effective temperatures, surface gravities and microturbulent velocities. Kurucz (1993) grid of atmosphere models is interpolated to obtain the fine grid. For all models the correlations between the abundances of iron, derived from the individual iron lines, and the equivalent widths, the energies of the lower levels and the wavelengths of these lines are calculated. Analysis of calculations for all models permit us to select the best parameters. These parameters should simultaneously satisfy 
the ionization balance, the zero correlations coefficients for the dependencies of iron abundances derived from individual lines vs. the equivalent widths, the potentials of low levels, the wavelengths of these lines and the scatter of individual iron abundance values should be minimal.

After a few iterations we were able to select the following values: $T_{\text {eff }}=7300 \mathrm{~K}, \log g=3.25, v_{\text {micro }}=3.8$ $\mathrm{km} \mathrm{s}^{-1}, \log \mathrm{N}(\mathrm{Fe})=7.44 \pm 0.08$ (in the scale $\log \mathrm{N}(\mathrm{H})=12$ ).

These values of effective temperature, surface gravity and microturbulance were used to plot Figs. 3 to 5: the dependencies of calculated abundances vs. the equivalent widths, the energies of low levels and the wavelengths of lines of neutral iron. The scattering of individual abundances is around \pm 0.15 dex. The uncertainties of atomic parameters, most significantly the oscillator strengths, are the main sources of the scatter.

Systematic errors due to the calibration of the scale of the effective temperatures, errors of equivalent widths, continuum placement, oscillator strengths, non-LTE effects and other reasons are possible. That is why we accepted the errors of effective temperature, surface gravity and microturbulent velocity to be equal to $100 \mathrm{~K}, 0.20$ dex and $0.3 \mathrm{~km} \mathrm{~s}^{-1}$ respectively as realistic values which include the internal errors of the method used only.

Systematic errors can significantly increase these numbers. The final uncertainties in the effective temperature, surface gravity and microturbulent velocity can be as high as $200 \mathrm{~K}, 0.4$ dex and $0.5 \mathrm{~km} \mathrm{~s}^{-1}$ respectively.

The selection of the error values of the derived parameters can be illustrated by Fig. 1 in our previous paper (Yushchenko et al. 2010). The last panel of this figure allows to estimate the internal precision of the method used. Note that the effective temperature, the surface gravity and the microturbulent velocity of $\rho \operatorname{Pup}\left(T_{\text {eff }}=6890 \mathrm{~K}, \log g=3.28, v_{\text {micro }}=4.1 \mathrm{~km} \mathrm{~s}^{-1}\right)$ are close to those of V1719 Cyg.

All calibrations of stellar effective temperatures and surface gravities are based on the investigations of individual bright stars. The scatter of the parameters derived by different methods for these bright stars can be even higher than the systematic errors accepted here for V1719 Cyg. For example the values of effective temperature of $\alpha$ Per published by different authors are in the range from $6750 \mathrm{~K}$ to $5967 \mathrm{~K}$ (Lee et al. 2006).

It is necessary to note that our two spectra were observed at the phases 0.74 and 0.01 of the main period. We found no significant changes in the equivalent widths and the profiles of spectral lines. The differencies of equivalent widths are not higher than several percents and there is no systematic change of equivalent widths between the two spectra. Additional observations are necessary to confirm or to exclude the possible variations of line profiles and equivalent widths of the lines at other phases. In this paper the second spectrum was accepted as the primary source for mea-

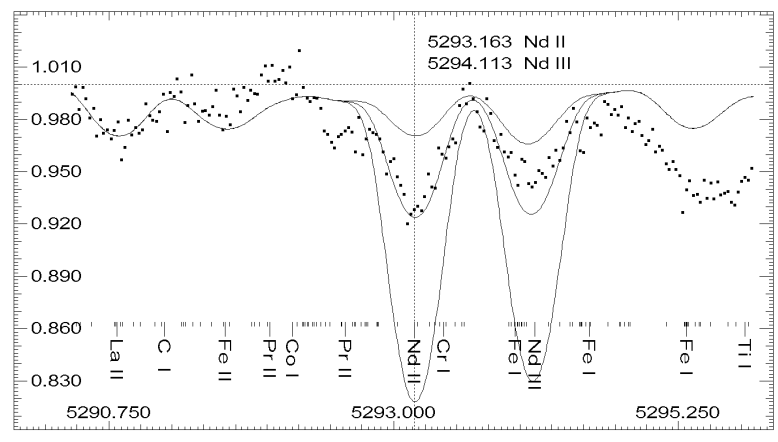

Fig. 6. - The observed (points) and synthetic (lines) spectra of V1719 Cyg in the vicinity of two lines of Nd. The axes are the wavelength in angstroms and relative fluxes. The positions of the spectral lines taken into account in the calculations are marked in the bottom part of the figure. For some of the strong lines the identifications are given. The position of the Nd II $5293.163 \AA$ line is marked by a vertical dotted line. The different synthetic spectra are calculated with solar abundance of $\mathrm{Nd}$ and to the abundances of $\mathrm{Nd}$ increased by 0.5 and 1.0 dex.

surements of the equivalent widths. That is why the values of effective temperature, surface gravity, microturbulence, and metallicity are found for the phase 0.01 of the main period. There are no significant differencies between the two spectra, but as the star is variable using the coadded spectrum cannot be justified.

Table 3 (available in the electronic version of the paper) contains the list of 89 lines of neutral iron and 30 lines of singly ionized iron. The columns are the ionization stages, wavelengths, oscillator strengths, energies of low levels, equivalent widths and derived abundances. For some of the lines, we calculated solar oscillator strengths using the Liege Solar Atlas (Delbouille et al. 1973) and Grevesse \& Sauval's (1999) solar model.

\section{DISCUSSION AND CONCLUSION}

The temperatures and surface gravities found from the spectra $\left(T_{\text {eff }}=7300 \pm 200 \mathrm{~K}, \log g=3.25 \pm 0.4\right)$ are in good agreement with those obtained from the photometry. Both Kurucz and MARCS calibrations (for zero phase: $T_{\text {eff }}=7225 \mathrm{~K}, \log g=3.27$ and $T_{\text {eff }}=7296$ $\mathrm{K}, \log g=3.41$ respectively) are within the error box of spectroscopic determination.

The metallicity of the star appears to be lower in comparison to the value found from photometry. The abundance of iron in the atmosphere of V1719 Cyg $\left(\log \mathrm{N}(\mathrm{Fe})_{V 1719 C y g}=7.44 \pm 0.08\right)$ is close to the solar one $\log \mathrm{N}(\mathrm{Fe})_{\odot}=7.45$ (Grevesse et al. 2007).

The comparison of observed spectrum with synthetic one, calculated with solar abundances of all elements show the possible overabundances of several iron group elements, yttrium, zirconium and lanthanides. This is illustrated in Fig. 6. This means that V1719 Cyg can 
be a metallic line (Fm) star. For Fm stars and also for Am and Ap stars, it is very usual that iron abundance is close to the solar value, but the heavier chemical elements, namely, r-, s-processes elements are overabundant. The overabundances of heavy elements can also explain the different values of metallicities, obtained from photometry and from high resolution spectroscopy. The detailed chemical composition needs additional investigation and will be the subject of the next paper.

\section{ACKNOWLEDGMENTS}

This work was supported by Korea Research Grant, 521-2008-C00122. We used data from NASA ADS, SIMBAD and NIST databases and we thank the teams and administrators of these projects.

\section{REFERENCES}

Alania, I. Ph. 1987, uvby $\beta$ Photometry of V1719 Cyg, Inf. Bull. Variable Stars 2994

Andreasen, G. K. 1983, Delta Scuti Variables. II - Comparison of Theoretical Evolution Sequences with Observational Data, A\&A 121, 250

Andreasen, G. K. 1987, Cepheids in the Magellanic Clouds. II - Search for Double Mode Cepheids in the LMC, A\&A 186,159

Antonello, E. 1991, The Infrared Spectral Region of Stars, Proceedings of the International Colloquium held in Montpellier, France, 16-19 October 1990. Cambridge University Press, p. 383

Antonello, E., Broglia, P., Conconi P., \& Mantegazza, L. 1986, Fourier Decomposition of the Light Curves of High Amplitude Delta Scuti and SX Phe Stars, A\&A, 169, 122

Bedolla, S., \& Pena, J. H. 1979, HD 200925, a New Short Period Variable Star, Inf. Bull. Variable Stars 1615

Bell, R. A., Paltoglou, G., \& Tripicco, M. J. 1994, The Calibration of Synthetic Colours, MNRAS, 268, 771

Clem, J. L., VandenBerg, D. A., Grundahl, F., \& Bell, R. A. 2004, Empirically Constrained Color-Temperature Relations. II. uvby, AJ, 127, 1227

Cox, A. N., Hodson, S. W., King, D.S. 1979a, Theoretical Periods and Masses of Double-Mode Dwarf Cepheids, ApJ, 228, 870

Cox, A. N., Hodson, S. W., King, D.S. 1979b, Theoretical Pulsation of Metallic-Line Stars, ApJ, 231, 798

Cox, A. N., McNamara B. J., \& Ryan, W. 1984, Observations and a New Interpretation for the Delta Scuti Variable Star VZ CANCRI, ApJ, 284, 250

Crawford, D. L. 1979, Empirical Calibrations of the UVBY, Beta Systems - Part Three - the A-Type Stars, AJ, 84, 1858

Dellbouille, L., Rolland, G., \& Neven, L. 1973, Photometric Atlas of the Solar Spectrum from $\lambda 3000$ to $\lambda 10000$, Institute de Astrophisique de Universitete de Liège

Fitch, W. S. 1981, L O, 1, 2, and 3 Pulsation Constants for Evolutionary Models of Delta-Scuti Stars, ApJ, 249, 218
Fuhr, J. R., \& Wiese, W. L. 2006, New Critical Compilations of Atomic Transition Probabilities for Neutral and Singly Ionized Carbon, Nitrogen, and Iron, J. Phys. Chem. Ref. Data, 35, 1669

Galazutdinov, G. A. 1992, Preprint 92, SAO RAN (Spets. Astrofiz. Obs. Ross. Akad. Nauk, Nizhnij Arkhyz)

Galazutdinov, G. A., Musaev, F. A., Krelowski, J., \& Walker, G. A. H. 2000, Narrow Diffuse Interstellar Bands: A Survey with Precise Wavelengths, ASP, 112, 648

Grevesse, N., Asplund, M., \& Sauval, A. J. 2007, The Solar Chemical Composition, Space Sciences Reviews, 130, 105

Grevesse, N., \& Sauval, A. J. 1999, The Solar Abundance of Iron and the Photospheric Model, A\&A, 347, 348

Gopka, V. F., Yushchenko, A. V., Mishenina, T. V., Kim, C., Musaev, F. A., \& Bondar, A. V. 2004, Atmospheric Chemical Composition of the Halo Star HD 221170 from a Synthetic-Spectrum Analysis, Astronomy Reports, 48, 577

Gustafsson, B., Bell, R. A., Eriksson, K., \& Nordlund, A. 1975, A Grid of Model Atmospheres for Metal-Deficient Giant Stars. I, A\&A, 42, 407

Hirata, R., \& Horaguchi, T. 1995, SIMBAD Catalog VI/69

Joner, S. B., \& Johnson, M. D. 1985, Photometric Properties of HD 200925, PASP, 97, 153

Johnson, M. D., \& Joner, S. B. 1986, Revised Photometric Properties for the Multiperiodic Variable HD 200925, PASP, 98, 581 (JJ86)

Kim, C., Jeon, Y.-B., \& Kim, S,-L. 2003, Differential TimeSeries CCD Photometry of BL Camelopardalis Revisited, PASP, 155, 755

Kim, C., Kim, S.-L., \& Sadakane, K. 1993, Pulsational Characteristics of V1719 Cygni with Peculiar Light Curve, JKAS, 26, 115

Kim, C., Yushchenko, A. V., Gopka, V. F., Dorokhova, T. N., Musaev, F. A., Kim, S.-L., Jeon Y.-B., Ibrahimov, M., \& Tarasov A. E. 2007, Chemical Composition and Differential Time-Series CCD Photometry of V2314 Ophiuchi: A New $\lambda$ Bootis-Type Star, AJ, 134, 926

Klein, B., Jura, M., Koester, D., Zuckerman, B., \& Melis, C. 2010, Chemical Abundances in the Externally Polluted White Dwarf GD 40: Evidence of a Rocky Extrasolar Minor Planet, ApJ, 709, 950

Kurucz, R. L. 1993, CDROMs No. 1-18 (Cambridge, MA, Smithsonian Astrophys. Obs.)

Kurucz, R. L. 1995, CDROM No. 23 (Cambridge, MA, Smithsonian Astrophys. Obs.)

Lee, B.-C., Galazutdinov, G. A., Han, I., Kim, K.-M., Yushchenko, A. V., Kim, J., Tsymbal, V., \& Park, M.G. 2006, A High Resolution Atlas of $\alpha$ Per from 3810 to $8100 \AA$, PASP, 118, 636

McNamara, D. H. 1985, The Rotational Velocities of the Dwarf Cepheids and Related Stars, PASP 97, 715

Mantegazza, L., \& Poretti, E. 1986, A Possible Second Period for HD 200925=V 1719 CYG, A\&A, 158, 389

Moon, T. T., \& Dworetsky, M. M. 1985, Grids for the Determination of Effective Temperature and Surface Gravity 
of B, A and F Stars Using uvby $\beta$ Photometry, MNRAS, 217,305

Morton, D. C. 2000, Atomic Data for Resonance Absorption Lines. II. Wavelengths Longward of the Lyman Limit for Heavy Elements, ApJS 130, 403

NIST atomic spectra database 2009, http://www.nist.gov/pml/data/asd.cfmnist_atomic spectra.html

Padalia, T. D., \& Gupta, S. K. 1982, A New Cepheid Variable, HD 200925, Ap\&SS, 81, 251 (PG)

Pena, J. H., et al. 2002, PASP, 114, 214

Piskunov, N., Kupka, F., Ryabchikova, T., Weiss, W., \& Jeffery, C. 1995, VALD: The Vienna Atomic Line Data Base., A\&AS, 112, 525

Poretti, E. 1984, UBV Photometry of the Short Period Pulsating Variable V1719 CYG, A\&AS, 57, 435 (EP)

Poretti, E., \& Antonello, E. 1988, The Peculiar Light Curves of Some Large Amplitude Delta SCT Stars, A\&A, 199, 191 (PA88)

Rutten, R. J., \& van der Zalm, E. B. J. 1984, Revision of Solar Equivalent Widths, Fe I Oscillator Strengths and the Solar Iron Abundance, A\&AS, 55, 143

Stellingwerf, R. F. 1972, Luminosity Variation in the OneZone Cepheid Model, A\&A, 21, 91

Tody, D. 1986, Instrumentation in Astronomy VI; Proceedings of the Meeting, Tucson, AZ, Mar. 4-8, 1986. Part 2 (A87-36376 15-35). Bellingham, WA, Society of PhotoOptical Instrumentation Engineers, p. 733

Watson, R. D. 1988, Contributing Factors to Flux Changes in Nonradial Stellar Pulsations, Ap\&SS, 140, 255

Yushchenko, A. V. 1998, in Proceedings of the 20th Stellar Conference of the Czech and Slovak Astronomical Institutes, Ed. by J. Dusek (ISBN 80-85882-08-6, Brno), p. 201.

Yushchenko, A. V., Gopka, V. F., Khokhlova, V. L., Musaev, F. A., \& Bikmaev, I. F. 1999, Atmospheric Chemical Composition of the "Twin" Components of Equal Mass in the CP SB2 System 66 Eri, Astronomy Letters 25, 453

Yushchenko, A., Gopka, V., Kim, C., Musaev, F., Kang, Y. W., Kovtyukh, V., \& Soubiran, C. 2005, The Chemical Composition of $\delta$ Scuti, MNRAS, 359, 865

Yushchenko, A. V., Dorokhova, T. N., Gopka, V. F., Kim, C., Lee, B.-C., Yushchenko, V. A., \& Doikov, D. N. 2005, The Atmosphere Parameters and Line Profile Variations of $\rho$ Puppis, 2010, JKAS, 43, 65 